\title{
New Electron Trap Might Help Quantum Computers
}

\section{Long-time trapping of a single electron could allow the particle to be used as an efficient quantum bit.}

By Philip Ball


uantum computers use quantum bits (qubits) that, in addition to encoding the states 1 and 0 , can be placed in combinations of the two. Several types of qubits are currently used, and now a research team has demonstrated that single electrons can be trapped effectively enough to

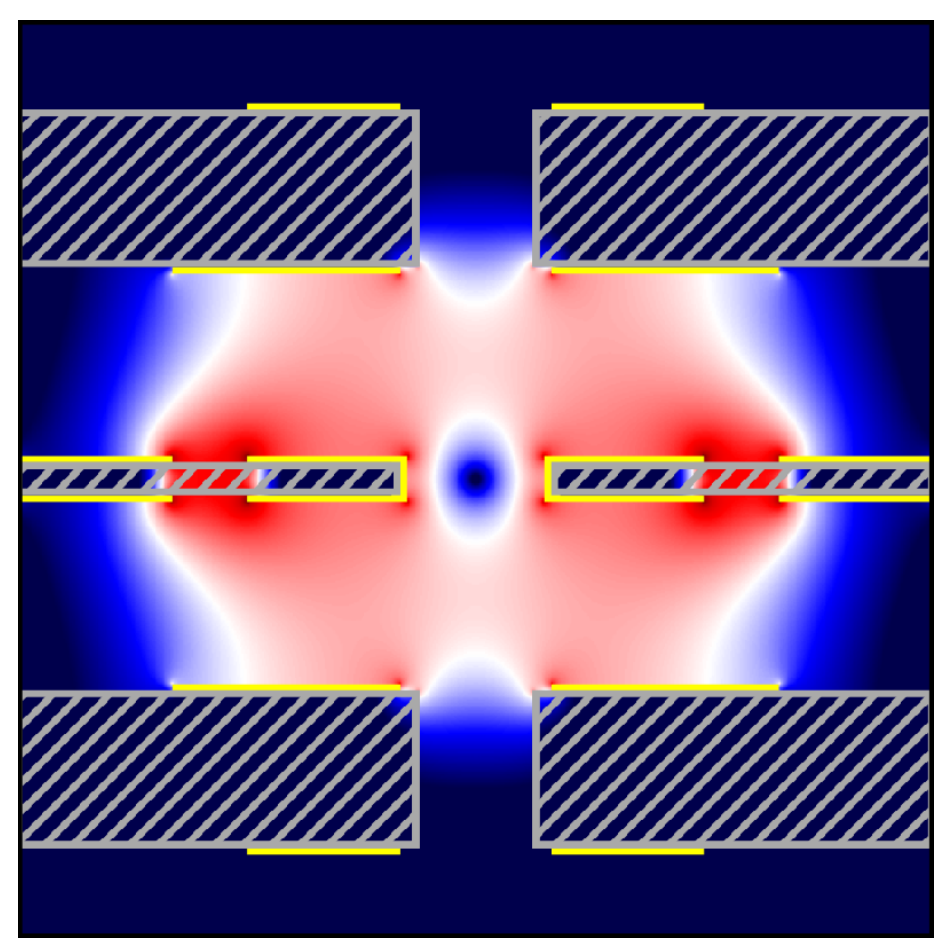

The eye of the trap. Theory predicts the potential energy surface for a slice through the three layers of circuit boards that make up the trap, showing a deep valley (central blue region, about a millimeter wide) to hold the electron. Gray hashed areas are the board cross sections, and yellow lines represent metal electrodes. Credit: C. Matthiesen et al. [1] potentially serve as a new, more efficient type of qubit [1]. The researchers trapped electrons for a record time of $1 \mathrm{~s}$. They say their results imply that electron qubits could be used to make faster quantum computers.

Qubits made from superconducting circuits or from single trapped ions-today's most practical qubit types-have inherent limitations, such as limits on the speed with which they can be switched from 1 to 0 . A trapped electron could act as a simple qubit based on its spin state-up or down. Hartmut Häffner of the University of California at Berkeley says that electrons should be able to switch 100 times faster than trapped-ion qubits and should be less susceptible to the environmental noise that degrades the performance of superconducting qubits. So, in principle, trapped electrons could yield fast and high-fidelity quantum computers.

However, trapping single electrons for long times isn't easy. It has been achieved before in configurations that would not be good for a qubit [2,3]-for example, using strong magnetic fields that would interfere with the qubit's operation. Single ion qubits, meanwhile, have usually been held in Paul traps, which use oscillating electric fields that can be generated by electrodes in a chip-based circuit [4].

To trap electrons this way, Häffner and colleagues created a microwave-frequency Paul trap that fits onto a three-layer, 5- by 10-cm circuit board containing a 1-mm-wide slot where the trapped electron floats. Starting with a low-energy electron helps to increase its lifetime in the trap. To generate such slow-moving (cold) electrons, the researchers adapted a procedure previously used to produce ions efficiently. The team hit a beam of calcium atoms with two lasers, one tuned to 


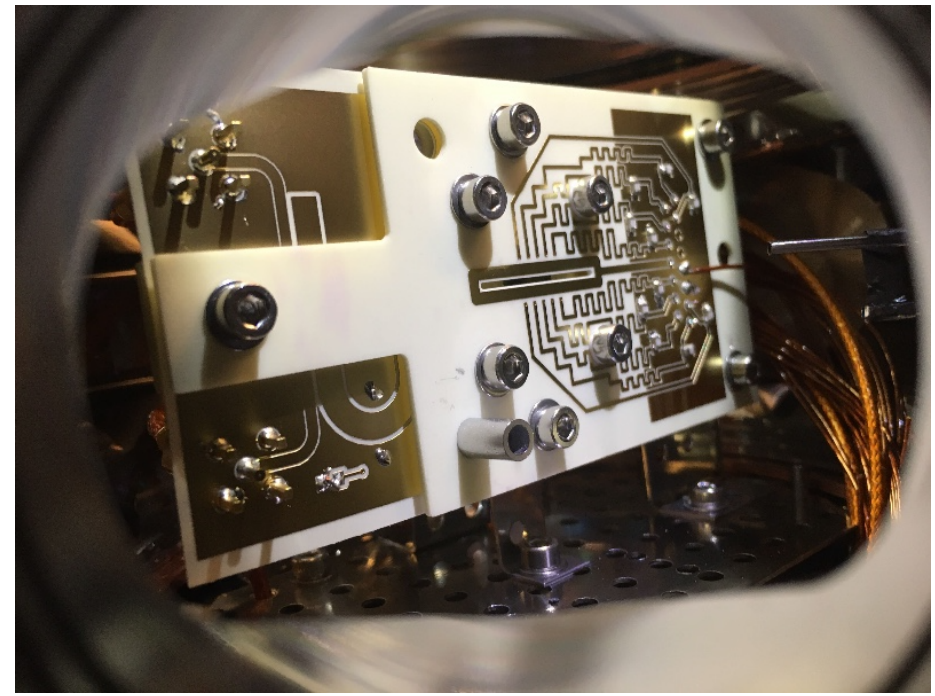

Electrons in the void. This credit-card-sized circuit board, shown in the experiment's vacuum chamber, houses an electric quadrupole trap that can hold individual electrons for more than a second. By entangling them and encoding quantum information in their spins, the electrons might eventually be used for quantum computing.

Credit: C. Matthiesen/Univ. of California, Berkeley

promote an electron to a highly excited state and the second tuned to provide just enough energy to exceed the atom's ionization threshold.

The researchers found that some electrons could be kept in their trap for more than a second at room temperature; they should last even longer at the low temperatures at which a quantum computer is likely to operate. On the other hand, around three-quarters of the electrons were lost within $0.1 \mathrm{~s}$. The researchers hope to improve the trapping efficiency once they have a better understanding of the loss mechanisms.

With a trapped electron qubit, readout of quantum information could be done using magnetic fields to sense the spins. Häffner and co-workers have already proposed a procedure of this sort, using oscillating magnetic fields to separate spin-up and spin-down electrons into streams that could be sensed by electrodes [5]. Putting that idea into practice will be one of the next steps, Häffner says, along with demonstrating a logic operation for a simple two-qubit quantum gate, also using magnetic fields to manipulate the spins.
"Nothing was really much more difficult than [using] a standard Paul trap," says Häffner. Team member Clemens Matthiesen admits that he shared some of the skepticism of others about whether electrons could be trapped for long enough. "But you can't know until actually trying the experiment," he says, "and I was somewhat shocked by how well it worked." One key to the success was using the calcium ionization technique to create electrons having sufficiently low energy, Häffner says.

"I like the results very much," says Ferdinand Schmidt-Kaler of the University of Mainz in Germany, who has been working with other kinds of electron traps. He agrees that electrons' high switching speed is an advantage over trapped ions and that using a chip-based trap-the next step in miniaturization of the new trap's circuitry-could make practical implementation easier.

Chris Monroe of the University of Maryland, College Park, who has worked with ion-based qubits in quantum-computing devices, says that the work is "compelling" but stresses that there are "lots of remaining challenges ahead" before it becomes a viable technology. Electrons have just one internal "knob" - their spin-which can't be manipulated simply with laser beams for read and write operations, as ion states can be. However, Häffner says that using chip-based magnetic operations for this, rather than lasers, could actually simplify the technology. Monroe adds that combining elements of electronic systems with those of trapped ions means that electron qubits "can hopefully adopt the best of both worlds."

Philip Ball is a freelance science writer in London. His latest book is How To Grow a Human (University of Chicago Press, 2019).

\section{REFERENCES}

1. C. Matthiesen et al., "Trapping electrons in a room-temperature microwave Paul trap," Phys. Rev. X 11, 011019 (2021).

2. D. Wineland et al., "Monoelectron oscillator," Phys. Rev. Lett. 31, 1279 (1973).

3. G. Koolstra et al., "Coupling a single electron on superfluid helium to a superconducting resonator," Nat. Commun. 10, 5323 (2019).

4. K. R. Brown et al., "Co-designing a scalable quantum computer with trapped atomic ions," npj Quantum Inf. 2, 1 (2016).

5. P. Peng et al., "Spin readout of trapped electron qubits," Phys. Rev. A 95, 012312 (2017). 